\title{
Control Issues of
}

\section{MEMS Nanopositioning Devices}

\author{
Y. Zhu, S. O. R. Moheimani, M. R. Yuce and A. Bazaei
}

\begin{abstract}
In this chapter, the control issues of Microelectromechanical System (MEMS) nanopositioning devices are introduced and discussed. The real-time feedback control of a novel micro-machined 1-degree-of-freedom (1-DoF) thermal nanopositioner with on-chip electrothermal position sensors is presented. The actuation works based on thermal expansion of V-shaped silicon beams. The sensing mechanism works based on measuring the resistance difference between two electrically biased identical silicon beams. The resistance difference varies with displacement. The heat conductance of the sensor beams varies oppositely with the position of the movable stage, resulting in different beam temperatures and resistances. A pair of position sensors are operated in differential mode to reduce low-frequency drift. The micromachined nanopositioner has a nonlinear static input-output characteristic. The electrothermal actuator has a dynamic range of $14.4 \mu \mathrm{m}$ and the electrothermal sensor has a low drift of $8.9 \mathrm{~nm}$ over 2000 seconds. An open-loop controller is first designed and implemented. It is experimentally shown that uncertainties result in unacceptable positioning performance. Hence, feedback control is required for accurate positioning. The on-chip displacement sensor is able to provide high-resolution displacement control. Therefore, a real-time closed-loop feedback control system is designed using a proportional-integral (PI) controller together with the nonlinear compensator used for the open-loop control system. The closed-loop system provides acceptable and robust tracking resolution for a wide range of set point values. The step response results show a positioning resolution of $7.9 \mathrm{~nm}$ and a time constant of $1.6 \mathrm{~ms}$ in a $10 \mu \mathrm{m}$ stroke. For triangular reference tracking, which is required in raster-scanned Scanning Probe Microscopy (SPM), the steady-state tracking error has a standard deviation of $20 \mathrm{~nm}$ within a wide range of $10 \mu \mathrm{m}$.
\end{abstract}

Keywords Microelectromechanical systems (MEMS), nanopositioning, electrothermal actuation, electrothermal position sensing, feedback control. 


\section{Introduction}

High precision nanopositioners have been extensively used in many applications such as scanning tunneling microscopy (STM) [1], atomic force microscopy (AFM) [2], and emerging ultrahigh density probe storage systems [3, $4,5,6,7]$. These nanopositioners typically have high positioning accuracy with a large dynamic range and a wide bandwidth, enabling fast and robust closedloop position control. Macro-scale nanopositioners can achieve nanometre-scale positioning resolution and accuracy, however they are relatively bulky and costly $[8,9,10,11]$. Microelectromechanical System (MEMS) nanopositioners have attracted significant interest recently because of their small size, low cost, fast dynamics and the emergence of applications such as probe-based data storage [12, $13]$, and scanning probe microscopy [14, 15, 16]. Closed-loop feedback control of these positioners is highly desirable if a high degree of displacement precision is required, and such a control system needs an accurate source of position information [17, 18, 19, 20]. However, many of the MEMS nanopositioners reported in the literature are not equipped with on-chip sensors due to the restrictions associated with micro-fabrication processes [21, 22, 23]. Thus, the inplane movements are often measured by laser reflectance microscopes [24, 25] or optical microscopes [26], making the footprint of the entire system fairly large. There are several exceptions in the literature, e.g., embedded on-chip capacitive and piezo-resistive displacement sensors were integrated in thermally and electrostatically actuated positioners in [27, 28, 29, 30]. Nevertheless, only openloop results were obtained and complex fabrication processes were required for electrical insulation between electrical heating and sensing circuits in [27] and for sidewall implementation of the piezo-resistors in [29]. In addition, limited sensing resolutions were achieved in [28] and [29]. Recently, a thermal sensing scheme was used in a probe-based storage device [31, 32]. Micro-heaters were used to measure the motion of a MEMS micro-scanner with resolution of less than $1 \mathrm{~nm}$. Compared to comb capacitive sensors, thermal sensors are much more compact and can be easily integrated with actuators in a MEMS device [33, 34, 35]. In [36, 37], off-chip electromagnetic coil actuators were adopted for scanner actuation along with the thermal sensing approach reported in [31, 32], where a complex mass-balanced structure was designed for vibration resistance purposes.

In this chapter, a novel electrothermal position sensor is integrated with a electrothermal actuator in the same MEMS chip without the need for inclusion of extra electrical insulation fabrication process as reported in [27], or assembling two chips as reported in [36, 37]. Although the electrothermal actuators consume more power compared with other MEMS actuators, for example electrostatic 
actuator and piezoelectric actuators, they do offer certain advantages. They can operate under low voltages, generate large forces, and enjoy a high degree of vibration resistance due to their stiff structures [38].

This chapter introduces a MEMS nanopositioner with on-chip electrothermal actuation and electrothermal sensing, which was micro-fabricated in a low-cost bulk silicon process. Measurement results show that the positioner has a dynamic range of $14.4 \mu \mathrm{m}$ and an open-loop bandwidth of $101 \mathrm{~Hz}$. To reduce the low frequency thermal drift, the sensors are operated in a pair and measured by a differential circuitry. The sensor was measured to have a small low-frequency drift of $8.9 \mathrm{~nm}$ over 2000 seconds. The on-chip displacement sensing enables a feedback control capability. A model of the positioner is derived and a proportional-integral (PI) feedback controller is implemented digitally in a dSPACE rapid prototyping system to investigate the closed-loop performance of the positioner. Open-loop and closed-loop performance of the system in tracking a step displacement were investigated. The closed-loop step response results show a steady-state RMS positioning error of $8.6 \mathrm{~nm}$, while the corresponding value of the open-loop seek operation can reach to $1180 \mathrm{~nm}$. High tracking performance was also achieved for a $10 \mathrm{~Hz}$ triangular reference by using a 2-DoF feedback control system consisting of a PI controller and a pre-filter.

The chapter is organized as follows: In section 2, the electrothermal positioning scheme and its modeling are introduced. Micro-fabrication process and design parameters are described in section 3. Section 4 presents the experimental setup and system characterization of the positioner. Open-loop static and dynamic responses are presented to verify that the positioner behaves as predicted. Section 5 presents the details of the open-loop and closed-loop control design. The tracking performances of step and triangle references are presented in this section. Section 6 concludes this chapter.

\section{Design and Modeling of Micromachined Nanopositioner}

\subsection{Design}

The proposed nanopositioner consists of electrothermal actuator and sensor on a single silicon chip. On-chip actuation and sensing enable a compact positioning system with small footprint and high resolution. Figure 1 illustrates the conceptual schematic view of the nanopositioner. The positioner stage is actuated by a chevron electrothermal actuator. To move stage, dc current is applied across the heaters to heat up the silicon beams. Compared to other actuation methods, 
for example, electrostatic actuation and piezoelectric actuation, the electrothermal actuators are simple to implement with low actuation voltage and large force. Thanks to the high stiffness of the structures, complex mass-balanced structures as proposed in [37] are not required to resist vibration. As shown in Figure 1, a pair of thin hot arms are designed to have an angle of $0.57^{\circ}$ with respect to each other in the $\mathrm{V}$-shaped electrothermal actuator. The operating voltage range is typically from 0 to $15 \mathrm{~V}$ in chevron actuators, which depends on the beam geometries. The displacement is proportional to the square of actuation voltage and the largest stroke of the actuator is limited by the buckling of the hot arms at high temperatures $\left(>700^{\circ} \mathrm{C}\right)[25]$.

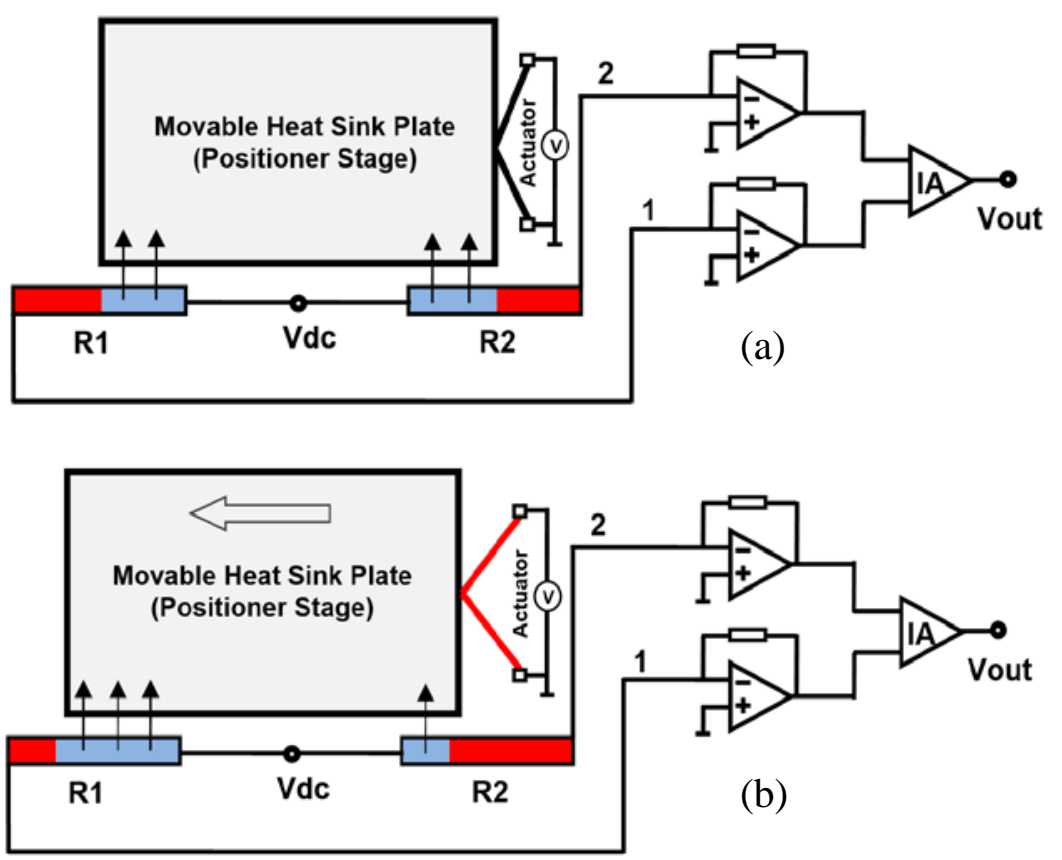

Figure 1 Schematic diagram of the nanopositioner with electrothermal actuator, electrothermal position sensor and differential amplifier: (a) before actuation; (b) after actuation. [39]

The position sensors are based on electrothermal principle. Two beam-shaped resistive heaters $R_{1}$ and $R_{2}$ are made from n-doped silicon (2 $\mu \mathrm{m}$ wide, $100 \mu \mathrm{m}$ long and $25 \mu \mathrm{m}$ thick). The positioner stage (rectangular plate) is placed close to the beam heaters with a $2 \mu \mathrm{m}$ air gap. Initially, the positioner stage is at the rest position before applying voltage across the actuator, as illustrated in Figure 1(a). At this position, the two edges of the sink plate are precisely aligned to the centre of the two thermal resistive sensors $R_{1}$ and $R_{2}$. To heat up the sensors, a dc voltage source $V_{d c}$ is applied to both sensors (heaters). Consequently, the heat generated in the resistive heaters is conducted through the $2 \mu \mathrm{m}$ air gap to the heat sink plate (positioner stage). At the rest position in Figure 1(a), the ambient condition for both sensors are identical, therefore, the dissipated heat fluxed out of the sensors are same. Thus, it equalizes the temperature and resistance of the two sensors. 
In Figure 1(b), a voltage is applied on the actuator beams to move the positioner stage towards left. As a consequence, the ambient condition is changed to be different. The heat fluxes dissipated out of the both sensors become different, resulting in a corresponding difference in the temperatures of both sensors. Also, the resistance is the function of temperature in silicon material. Therefore the resistance of sensor $R_{1}$ and sensor $R_{2}$ will be different. Thus, the positioner stage's displacement can be sensed by measuring the resistance changes between the two sensors. With a fixed dc voltage $V_{d c}$ applied to both sensors, the differential changes of the resistance result in current variations in the beam resistors because of Ohm's law. The currents are then converted to an output voltage using a pair of trans-impedance amplifiers and an instrumentation amplifier. To suppress the common-mode noise, the gains of these two trans-impedance amplifiers must be well matched by adjusting the feedback resistance of the trans-impedance amplifiers. Employing the differential topology allows the sensor output to be immune from undesirable drift effects due to changes in ambient temperature or aging effects.

\subsection{Modeling}

This section presents a large-signal analysis for anticipation of temperature and resistance values of the heaters in terms of a pre-specified displacement and bias voltage. We adopt a lumped parameter approach and static (time-invariant) conditions to simplify the analysis [40]. Each sensing resistor is regarded as an infinitesimal solid element with no internal thermal convection. With this assumption, the resistor temperature is presented by a scalar variable rather than a spatial distribution, which would require solving a complicated boundary value PDE problem. In addition, using this assumption, we can combine a number of heat transfer coefficients between the object and various parts in its surrounding such as heat sink, air gap, and conducting pads, into an overall thermal resistance coefficient. Applying Newton's law of cooling, the transfer of the heat from the object to air is mainly governed by thermal conduction in a thin air layer beside the object instead of thermal convection. Although thermal convection can participate in the transfer of heat outside the foregoing thin layer, its overall affect in stationary conditions can be modeled as an equivalent thermal resistance between the object and air. In this way, each sensing resistor has a uniform temperature distribution. We now proceed to a lumped parameter analysis for temperature prediction of heaters. 


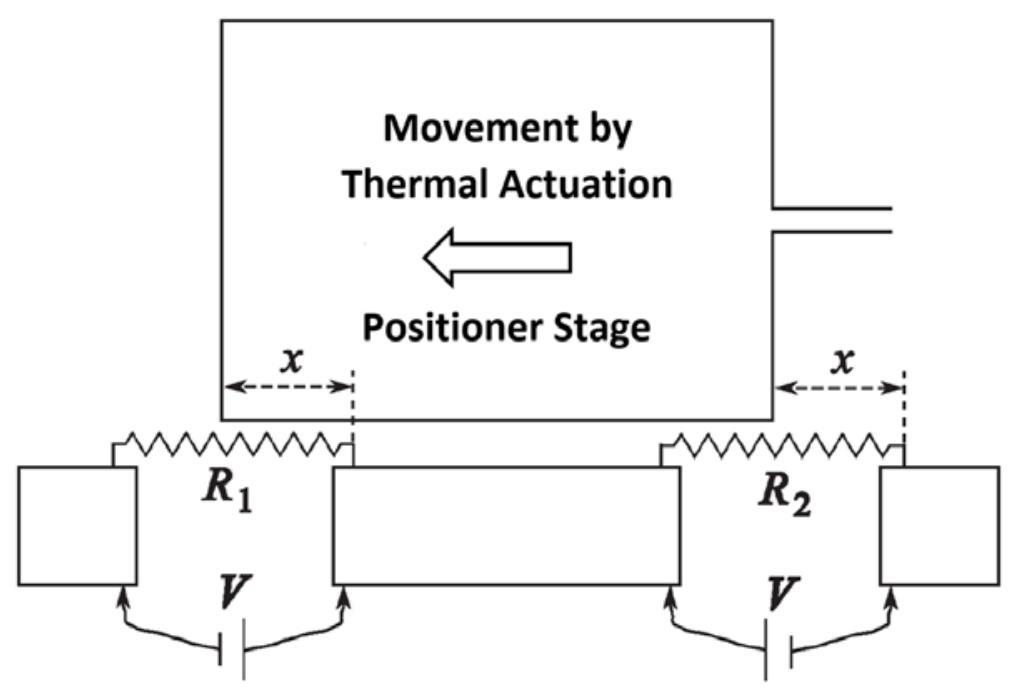

Figure 2 Schematic diagram of the biased electrothermal position sensors. [39]

Assuming a constant temperature coefficient $\alpha\left(1 /{ }^{\circ} \mathrm{C}\right)$, the electrical resistance $R$ of an infinitesimal resistor at temperature $T$ is described by $R_{o}\left[1+\alpha\left(T-T_{o}\right)\right.$ ], where $T_{o}$ and $R_{o}(\Omega)$ describe the ambient temperature and the resistance value at room temperature, respectively. Let the overall thermal resistance between the resistor and the surrounding area, including the thermal conduction through its electrical wiring to a voltage source, be $K^{-1}\left({ }^{\circ} \mathrm{C} / \mathrm{W}\right)$. Assuming a constant bias voltage $V$ across the resistor and a stationary condition, the electrical power delivered to the resistor should be equal to the heat power delivered by the resistor to the ambient area:

$$
\frac{V^{2}}{R_{o}\left[1+\alpha\left(T-T_{o}\right)\right]}=K\left(T-T_{o}\right)
$$

Thanks to the simplifying assumption that constant $\alpha$ does not vary with the temperature, we can obtain a closed-form solution for the temperature $T$ from Equation (1). Hence, the temperature and electrical resistance of the resistor at bias voltage $V$ are determined by the following expressions:

$$
R=R_{o} \frac{1+\sqrt{1+\frac{4 \alpha V^{2}}{R_{o} K}}}{2}
$$




$$
T=T_{o}+\frac{\sqrt{1+\frac{4 \alpha V^{2}}{R_{o} K}}-1}{2 \alpha}
$$

Before applying Equations (2) and (3) to the heaters in Figure 2, we need to introduce individual heat resistances $K_{1}^{-1}$ and $K_{2}^{-1}$ to the ambient area for each electric resistors $R_{1}$ and $R_{2}$, respectively. A horizontal displacement of the heat sink plate in the left direction will increase the heat conductance of the left heater, whilst reducing that of the right heater. These monotonic variations of the heat conductance values with displacement can roughly be modeled by the following linear relationships:

$$
\begin{aligned}
& K_{1}\left(\ell \varnothing=K_{\text {min }}+\left(K_{\text {max }}-K_{\text {min }}\right) \ell^{\prime},\right. \\
& K_{2}(\ell)=K_{\text {min }}+\left(K_{\text {max }}-K_{\text {min }}\right)(1-\ell \zeta)
\end{aligned}
$$

where $\mathscr{L} \in X / L$ describes a dimensionless position variable, $L$ is the heater length, and $K_{\min ^{-1}}$ and $K_{\max }{ }^{-1}$ represent the maximum and minimum overall heat resistance of each heater, respectively. We can now use Equations (2) and (3) to predict the stationary values of temperature and resistance for each heater:

$$
\begin{aligned}
& R_{i}(\ell, O V)=R_{o} \frac{1+\sqrt{1+\frac{4 \alpha V^{2}}{R_{o} K_{i}(\ell x)}}}{2}, \\
& T_{i}(\ell, O V)=T_{o}+\frac{\sqrt{1+\frac{4 \alpha V^{2}}{R_{o} K_{i}(\ell)}}-1}{2 \alpha}, i \in\{1,2\}
\end{aligned}
$$

where $R_{o}$ is value of unbiased heater electrical resistance at room temerature. When the heat sink displacement is zero $(\tilde{x}=0.5)$, we have assumed an identical heat conductance $K_{1}=K_{2}=\left(K_{\max }+K_{\min }\right) / 2$ for the heaters. Hence, the sensing resistors are also identical when the displacement is zero. In this way, the output voltage in Figure 1 is proportional to $R_{1}^{-1}-R_{2}^{-1}$. This result is used in the following simulation to convert the amount of heat sink displacement into a dimensionless variable $R_{o}\left(R_{1}^{-1}-R_{2}^{-1}\right)$, which is proportional to the output voltage in Figure 1. 
Considering the values reported in Table 1 for model parameters and applying Equations (4) and (5), the stationary temperature, resistance, and sensor output values as a function of heat sink displacement are obtained for each sensing heater as depicted in Figure 3(a)-(c), respectively. In spite of the nonlinear variation of the individual resistance values with displacement, the sensor output demonstrates a highly linear relationship with the displacement.

Table 1 Electrothermal Sensor Parameters and Values for Simulation

\begin{tabular}{|c|c|}
\hline Parameters & Values \\
\hline$\alpha\left(\mathrm{K}^{-1}\right)$ & $3.7822 \times 10^{-4}$ \\
\hline $\mathrm{R}_{0}(\Omega)$ & 390 \\
\hline $\mathrm{T}_{0}\left({ }^{\circ} \mathrm{C}\right)$ & 27 \\
\hline $\mathrm{K}_{\min }(\mathrm{W} / \mathrm{K})$ & $8 \times 10^{-5}$ \\
\hline $\mathrm{K}_{\max }(\mathrm{W} / \mathrm{K})$ & $16 \times 10^{-5}$ \\
\hline $\mathrm{V}(\mathrm{V})$ & 6 \\
\hline
\end{tabular}

(a)

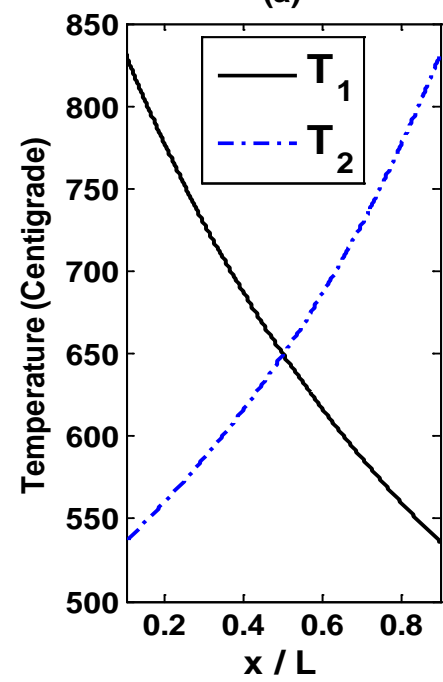

(b) Resistances

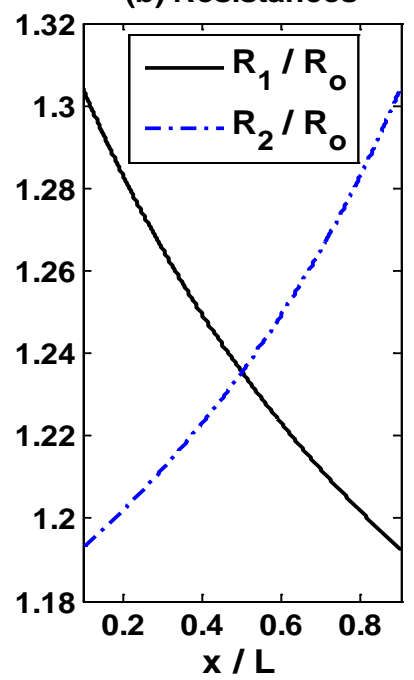

(c) Sensor output

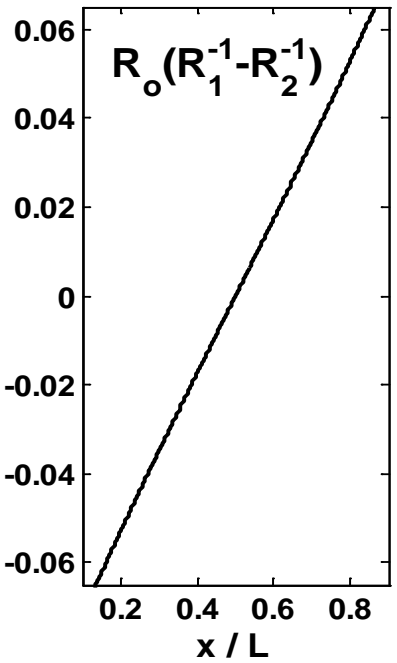

Figure 3 Simulated steady-state values versus displacement for (a) resistor temperatures, (b) resistance values, and (c) normalized sensor output. [39] 


\section{Microfabrication}

The device was fabricated in a commercial Silicon-On-Insulator (SOI) Micromachining process in a MEMS foundry (MEMSCAP) [41]. This process allows fabrication of silicon structures with a fixed $25 \mu \mathrm{m}$ of thickness and a minimum $2 \mu \mathrm{m}$ of width and gap. The final device structure is illustrated in Figure 4, and the fabrication process is briefly described as follows:

1. Microfabrication starts with a highly doped n-type $25 \mu \mathrm{m}$ silicon device layer, on which metal pads (20 nm $\mathrm{Cr}$ and $500 \mathrm{~nm} \mathrm{Au}$ ) are deposited by e-beam evaporation and patterned through lift-off process. The metal pads are to provide ohmic contact for probes and/or bonding wires.

2. Silicon is lithographically patterned to define the structures. Then deep reactive ion etch (DRIE) is applied from the front side of the wafer to define both the anchored and movable features of the structure.

3. A protective polyimide layer is applied to the top surface of the silicon layer to protect the silicon structures during the following etching process.

4. Another deep reactive ion etch (DRIE) is applied from the back side of the wafer to etch completely through the substrate in defined areas, stopping on the buried oxide layer. Then a trench underneath the movable structures is created.

5. The exposed buried oxide is removed using a wet oxide etch process (HF etching).

6. The polyimide coat on the front side is removed by oxygen plasma, thereby allowing the movable structure to be fully released. The front side oxide layer is removed using a vapor HF process. Then a large contact metal pad (i.e. electrical grounded contact) is patterned on the substrate to reduce parasitics.

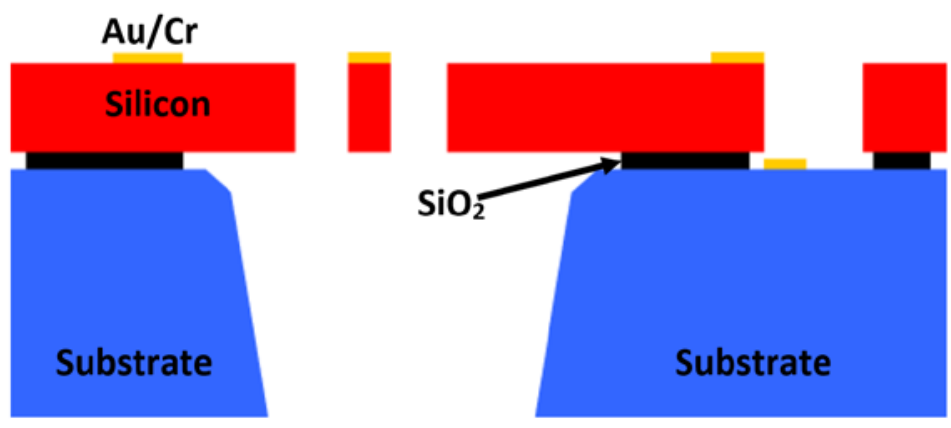

Figure 4 SOIMUMPs microfabrication structures from MEMSCAP [41]

The Scanning Electron Microscope (SEM) image of the whole device and a close-up section of it are provided in Figure 5. 


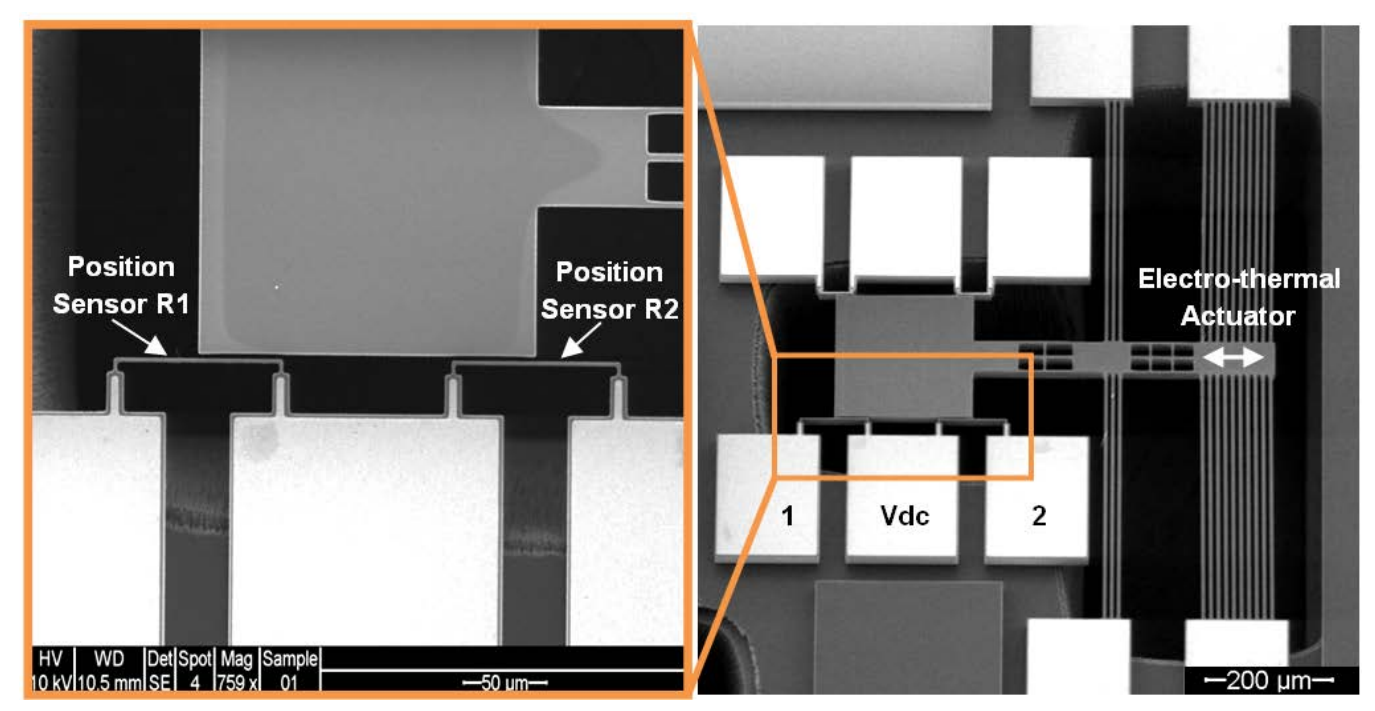

Figure 5 SEM images of the micromachined nanopositioner. The close-up section shows the details of a pair of electrothermal sensors, and positioner stage as a heat sink for the sensors [39]

To reduce the thermal coupling effects from the electro-thermal actuators to thermal sensors, a number of holes were embedded in the centre shuttle between actuators and the heat sink plate. The holes are expected to improve the thermal convection, thereby thermally insulating the heat sink plate from actuators. The suspension beams help the thermal insulation as well, due to the heat transfers to the substrate through the suspension beams and its anchors. Table 2 summarizes the device design parameters and measured beam resistance.

Table 2 Design Parameters and Values

\begin{tabular}{|c|c|}
\hline Parameters & Values \\
\hline Thermal actuator beam width $(\mu \mathrm{m})$ & 5 \\
\hline Thermal actuator beam length $(\mu \mathrm{m})$ & $2 \times 400$ \\
\hline Thermal actuator beam angle $($ degree$)$ & 0.57 \\
\hline Thermal actuator beam quantity $($ pair) & 10 \\
\hline Suspension beam width $(\mu \mathrm{m})$ & 5 \\
\hline Suspension beam length $(\mu \mathrm{m})$ & 400 \\
\hline Suspension beam angle $($ degree$)$ & 0 \\
\hline Suspension beam quantity $($ pair) & 3 \\
\hline Thermal sensor beam width $(\mu \mathrm{m})$ & 2 \\
\hline Thermal sensor beam length $(\mu \mathrm{m})$ & 100 \\
\hline Heat sink plate dimension $(\mu \mathrm{m} \times \mu \mathrm{m})$ & 2 \\
\hline Device thickness $(\mu \mathrm{m})$ & $200 \times 200$ \\
\hline Gap between thermal sensor and heat sink plate $(\mu \mathrm{m})$ & 25 \\
\hline Measured resistance of thermal sensor R1 at room temperature $(\Omega)$ & 388.9 \\
\hline Measured resistance of thermal sensor R2 at room temperature $(\Omega)$ & 391.6 \\
\hline Measured resistance of electro-thermal actuator at room temperature $(\Omega)$ & 116 \\
\hline
\end{tabular}




\section{Experimental Characterization}

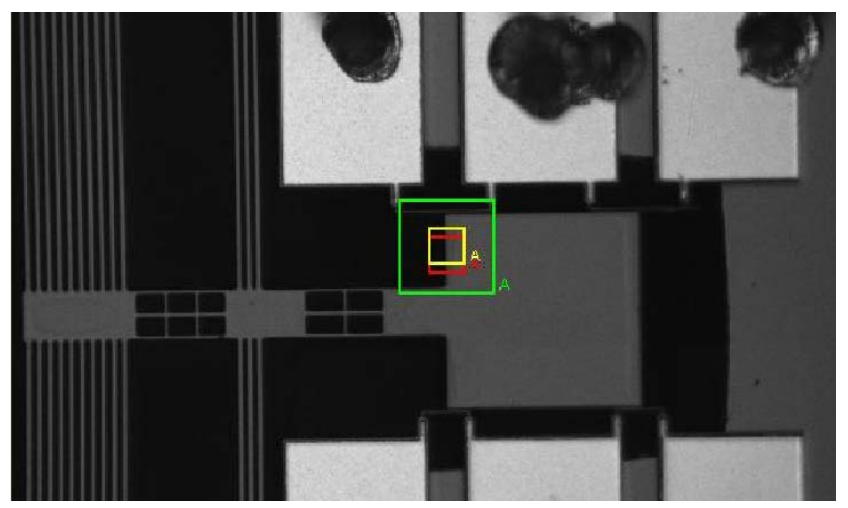

Figure 6 Displacement measurement using Polytec ${ }^{\mathrm{TM}}$ Planar Motion Analyzer (PMA-400, Polytec GmbH)

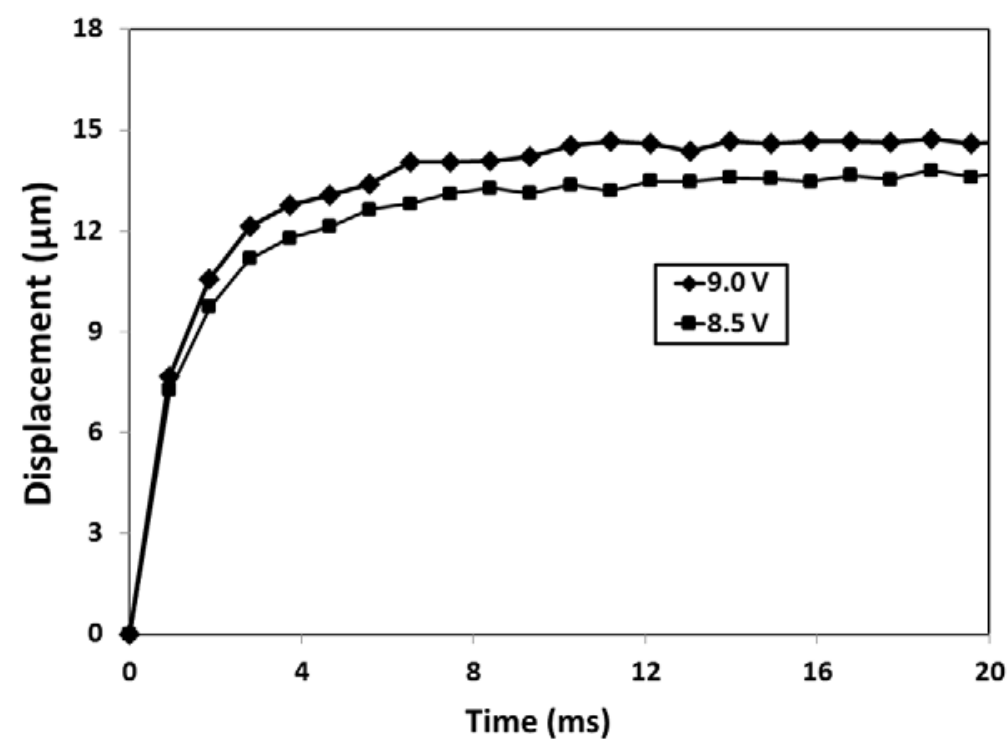

Figure 7 Time response of the electrothermal actuator with step actuation voltages of $8.5 \mathrm{~V}$ and $9.0 \mathrm{~V}$.

The displacement of the nanopositioner was calibrated using a Polytec ${ }^{\mathrm{TM}}$ Planar Motion Analyzer (PMA-400, Polytec GmbH). To accurately characterize the in-plane displacement of the positioner stage, digital image capturing and analysis methods were utilized. The PMA has an ordinary speed camera with a limited exposure time of $82 \mathrm{~ms}$, however it can measure periodic processes at frequencies as high as $1 \mathrm{MHz}$ by using the stroboscopic principle [42]. Step response is essential to characterize the dynamic performance of the positioner. Time constant and displacement can be obtained through step response measurement. Therefore, step voltages were applied to the actuator periodically, and the PMA recorded the displacement time response in each period, as shown in Figure 6. The step time response of the actuator has been characterized using 
different actuation voltages of $8.5 \mathrm{~V}$ and $9.0 \mathrm{~V}$ to exam the step displacement and time constant. As illustrated in Figure 7, after $20 \mathrm{~ms}$ of actuation, the positioner stage reached the stable positions of $13.6 \mu \mathrm{m}$ and $14.4 \mu \mathrm{m}$ with $8.5 \mathrm{~V}$ and $9.0 \mathrm{~V}$ step actuation voltages respectively. The time constant is approximately $1.6 \mathrm{~ms}$ for both cases.
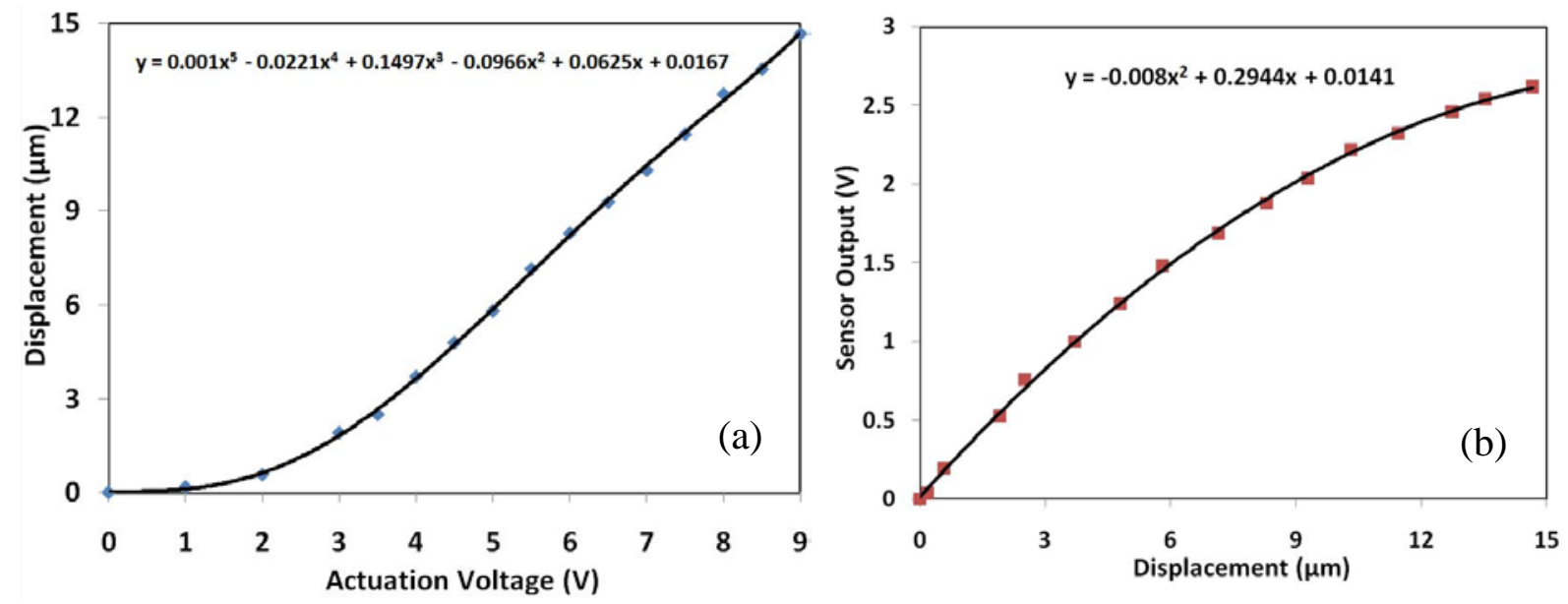

Figure 8 Experimental calibration curves: (a) displacement versus actuation voltage for actuator; (b) output voltage versus displacement for sensor.

To control the positioner stage with high resolution, the static characterization of the actuator and the sensor must be implemented accurately. Particularly, two calibration curves are required: displacement versus actuation voltage curve for the actuator and output voltage versus displacement curve for the sensor. Accurate displacement measurement is crucial for the above two calibration curves. Therefore, Planar Motion Analyzer (PMA) is utilized to measure the inplane displacement. The static displacement versus actuation voltage measured data with a fitted fifth order polynomial is illustrated in Figure 8(a). With the actuation voltage of $9 \mathrm{~V}$, the electrothermal actuator can achieve a maximum displacement of $14.4 \mu \mathrm{m}$, which is matched well with the step response measurement in Figure 7. Due to the nature of the thermal expansion, the actuator can only move forward in one direction after application of a voltage. However, the actuator can be biased to an initial position by a dc offset voltage, such that it can move forward or backward by increasing or decreasing the applied voltage. For instance, for a $10 \mu \mathrm{m}$ travel range with bi-directional movement, the actuator can be actuated by a $4.5 \mathrm{~V}$ dc voltage to the middle position $(5 \mu \mathrm{m})$ of the desired range.

To calibrate the position sensor, the instrumentation amplifier outputs were recorded at every actuation voltage, as illustrated in Figure 8(b). The sensors were biased with $6 \mathrm{~V}$ and the instrumentation amplifier gain was set at $90.3 \mathrm{~V} / \mathrm{V}$. With the bias voltage of $6 \mathrm{~V}$, a power of $60 \mathrm{~mW}$ is consumed in each sensor and the 
sensitivity is $0.27 \mathrm{mV} / \mathrm{nm}$ with a displacement up to $6 \mu \mathrm{m}$. As shown in Figure 8(b), the sensitivity drops when the displacement is more than $6 \mu \mathrm{m}$. A possible reason is that the temperature distribution is not uniform in the thermal sensor beams. Because of the heat loss at the anchors of the sensor beams, the centre of the beam is hotter than its two ends, as described in [43].

To visualize the temperature distribution, a $10 \mathrm{~V}$ dc voltage was applied to the left sensor in Figure 9. Visible light was observed in the centre of the silicon beam while the two ends of the sensor beams have no light. The right sensor is dark because no voltage was applied to it for the comparison purpose. It was observed that $10 \mathrm{~V}$ is safe for the silicon beam without damage. However, the bias voltage should be far less than $10 \mathrm{~V}$ for the sensor's reliability reason. Therefore, the sensor is calibrated with $6 \mathrm{~V}$ bias voltage.

It will be of interest to investigate the effect on the sensor sensitivity from the bias voltage. The relationships between positioner displacement and sensor output at various sensor biases are illustrated in Figure 10. The sensitivity can be increased by increasing the sensor bias voltage. However, the nonlinear nature of the response could not be eliminated by increasing the bias voltage.

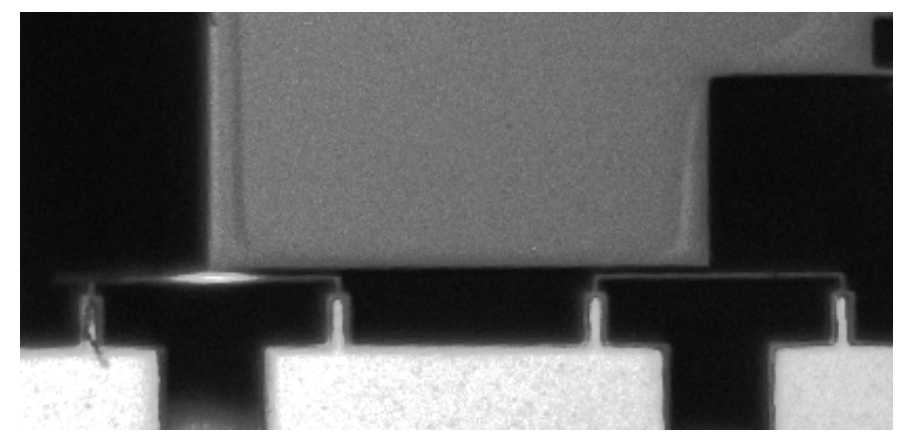

Figure 9 Optical micrograph of the position sensor's illumination with an applied voltage of $10 \mathrm{~V}$ to the left silicon beam. No voltage was applied to the right sensor for comparison purpose. [39] 


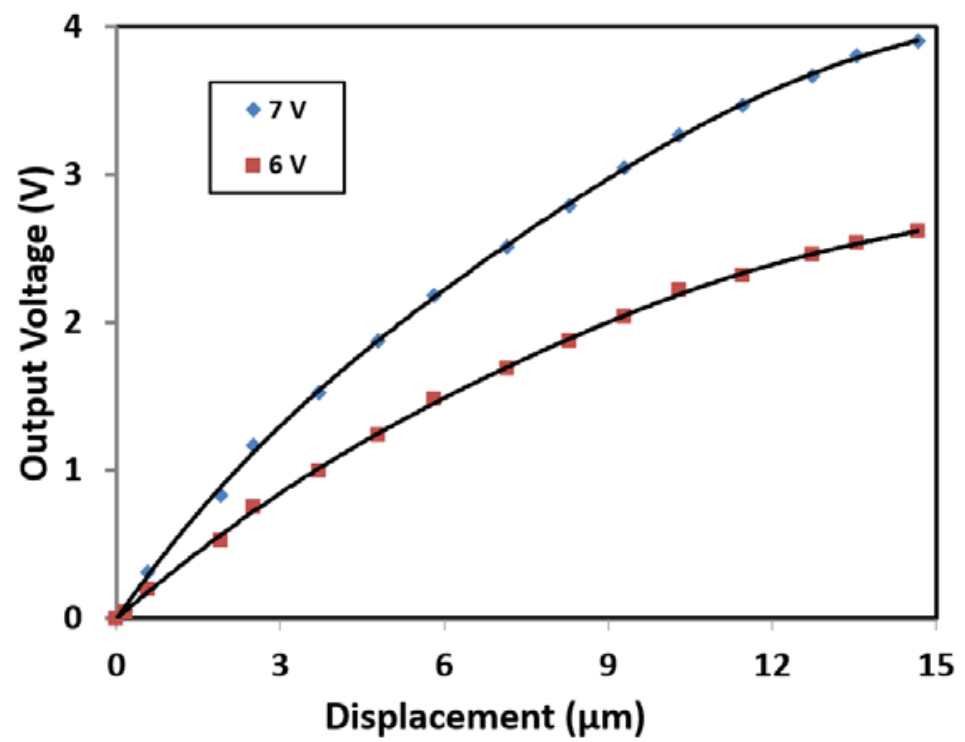

Figure 10 Output voltage versus displacement with different sensor bias voltages of 6 and 7 V. [39]

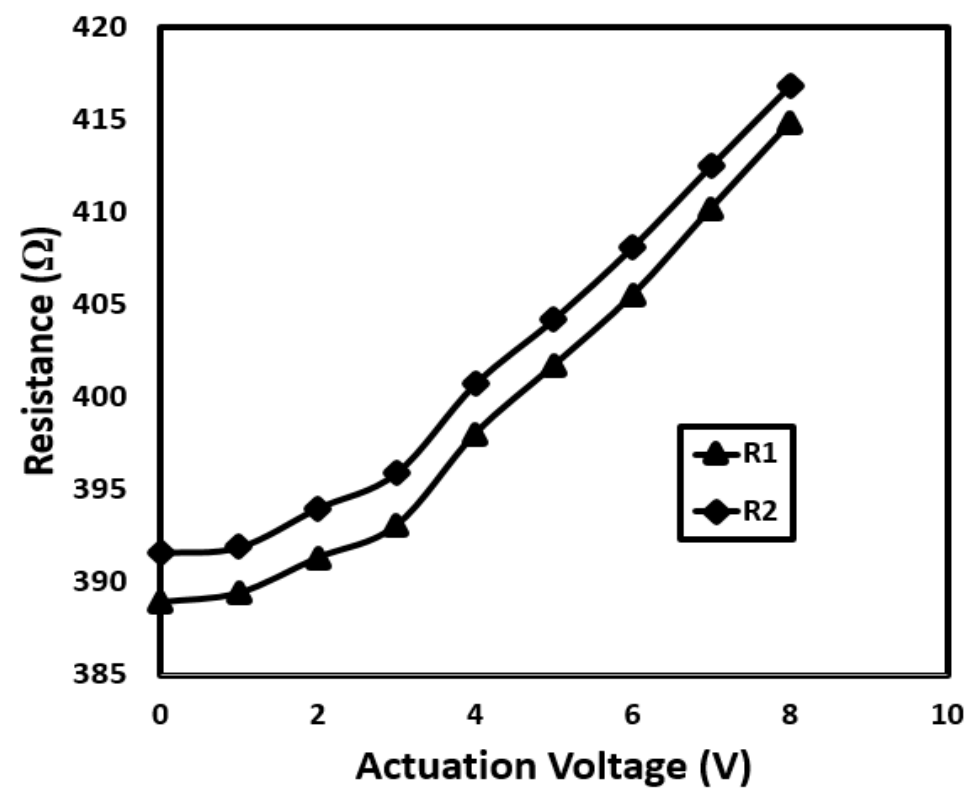

Figure 11 Measured resistance versus voltage with no bias voltage applied to the sensor pair.

As actuator and sensor are on a single silicon chip, we cannot assume that the displacement does not have any effect on the temperature of the area around the sensor resistors. To investigate the thermal coupling from actuator to sensor, the sensor resistances were measured using multimeter without applying an external bias voltage. As shown in Figure 11, it is observed that the un-biased sensor resistances increase about 6.4 percent for a $12 \mu \mathrm{m}$ displacement, just due to transfer of heat from actuator to sensor. The difference between the two curves in Figure 11 is because of the variations and errors in beam dimensions during microfabrication. 


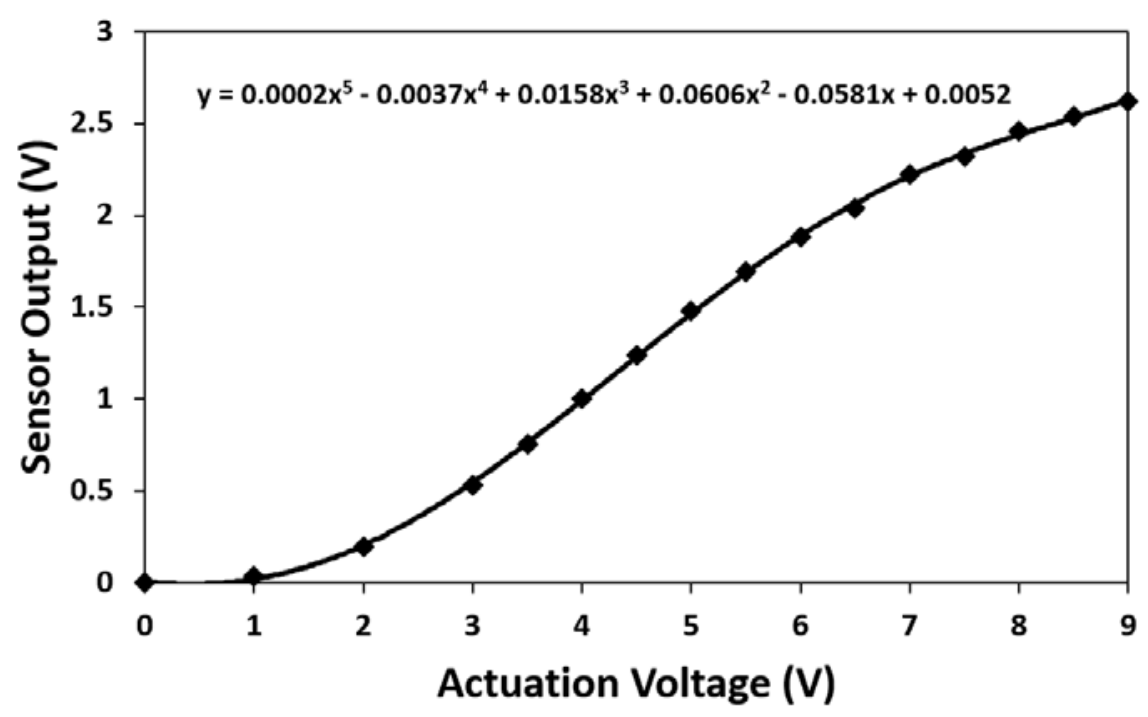

Figure 12 Experimental calibration curve: actuation voltage versus sensor output votage for the overall system including actuator and sensor.

Dynamic characterization is crucial for control implementation. HP35670A spectrum analyzer was utilized to characterize the positioner's dynamic performance. To characterize the frequency response, the nanopositioner can be regarded as an input-output system, which is connected to the spectrum analyzer. The input actuation voltage versus sensor output voltage curve can be obtained from the measured data in Figure 8. The fitted fifth order polynomial calibration curve is illustrated in Figure 12. To drive the actuator at high frequency, a dc voltage of $4.5 \mathrm{~V}$ superimposed with an ac voltage of $1 \mathrm{~V}$ was applied to the electrothermal actuator. Swept sinusoidal measurements were carried out from 1 $\mathrm{Hz}$ to $51.2 \mathrm{kHz}$ by applying the input voltage to the actuator and sensing from the on-chip thermal sensors. The frequency response, illustrated in Figure 13, shows that the open-loop bandwidth of the positioner is $101 \mathrm{~Hz}$, which is relatively low compared to the thermal structures fabricated by PolyMUMPs [44]. The reason is because of the fact that the heat transfer to surrounding air in SOIMUMPs is much slower than that to the substrate in PolyMUMPs. However, the bandwidth of $101 \mathrm{~Hz}$ is satisfactory for some positioning applications. 


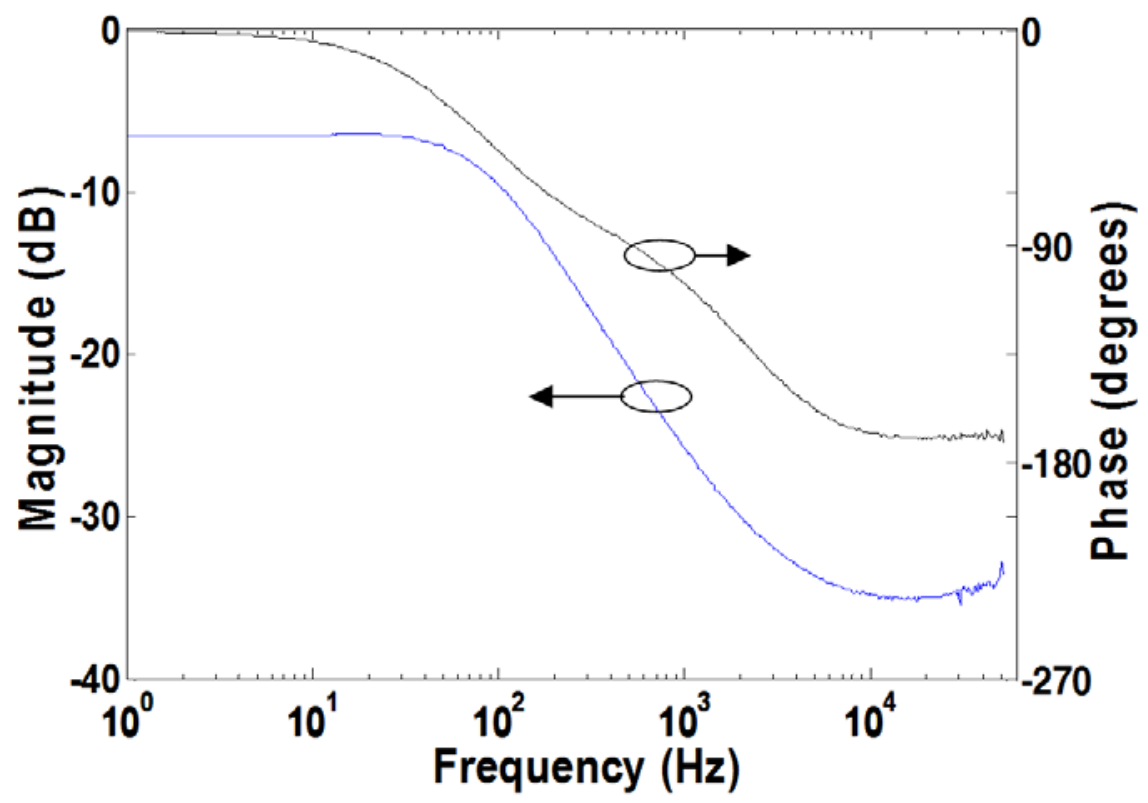

Figure 13 Measured open-loop frequency response of the MEMS nanopositioner using HP35670A spectrum analyzer.

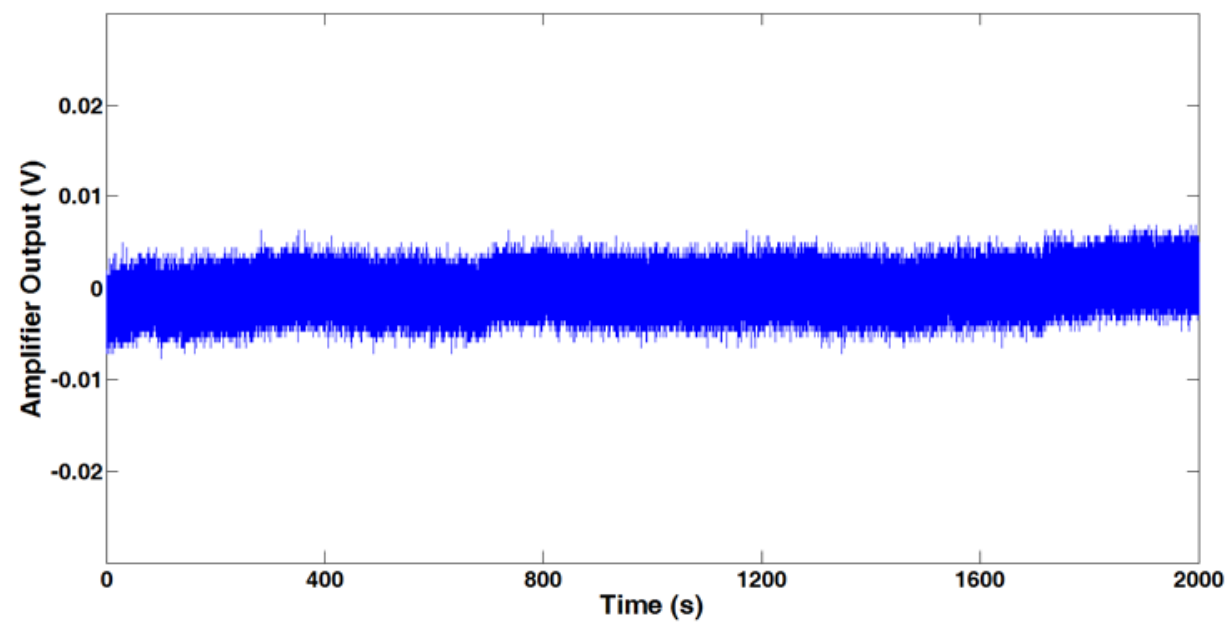

Figure 14 Experimental results of sensor drift (open-loop) over 2000 seconds.

Figure 14 shows the open-loop sensor drift when the positioner is at rest, which was measured at the output of the instrumentation amplifier over a period of 2000 seconds under normal laboratory conditions. Low frequency drift can be calculated by the moving average of the measured data. Thanks to the differential sensing of the sensor pair, the open-loop amplifier output has a low drift of 2.4 $\mathrm{mV}$ over 2000 seconds, which corresponds to $8.9 \mathrm{~nm}$ displacement. 


\section{Control Implementation}

Having known the static and dynamic parameters of electrothermal actuators and sensors, proper control strategies can implemented to achieve high positioning resolution and stability for the MEMS nanopositioner. In this section, open-loop and closed-loop position control strategies are investigated to control the MEMS positioner to have a positioning resolution in nanometre range. A dSPACE-1103 rapid prototyping system was used for real time implementation of the controllers and data acquisition. The dSPACE system is fully programmable from the Simulink ${ }^{\circledR}$ block diagram in Matlab environment.

\subsection{Open-loop control}

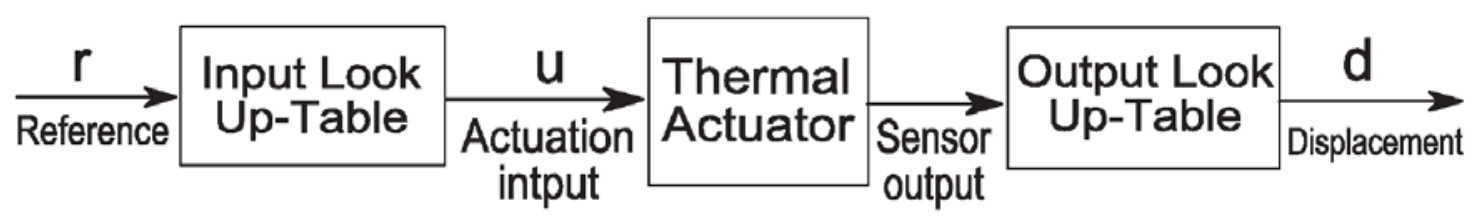

Figure 15 Block diagram of the open-loop control system including input and output look up tables, reference, electrothermal actuator and sensor. [39]

The block diagram of the open-loop control system is shown in Figure 15. Accurate look-up-tables are required for fast computation of static nonlinear input-output mappings. For a specific input value to the block, it generates an output value based on interpolation or extrapolation among the recorded data values. The look-up-table at the input of the electro-thermal actuator converts the reference signal in micrometres to an appropriate actuation voltage, as illustrated in Figure 16(a), which was obtained using Planar Motion Analyzer as described in Section 5. However, the number and precision of measured data points are not enough to provide a smooth curve. Therefore, a fifth order polynomial was fitted to the measured data in Figure 16(a) to generate the data for the input look-uptable. In similar way, the look-up-table at the output of the electrothermal actuator was obtained to convert the sensor output voltage to displacement in micrometres, as shown in Figure 16(b). For a sensor bias voltage of 7 volts, a third order polynomial was fitted to the measured data points in Figure 10 to generate smooth and monotonic data for the output look-up-table. To avoid the infinite slope of the curve around zero displacement in Figure 16(a), the origin for displacement 
was shifted to +1 micrometer. Then both input and output look-up-tables were input to Simulink to control the electrothermal actuator.

(a) Input Look-Up-Table

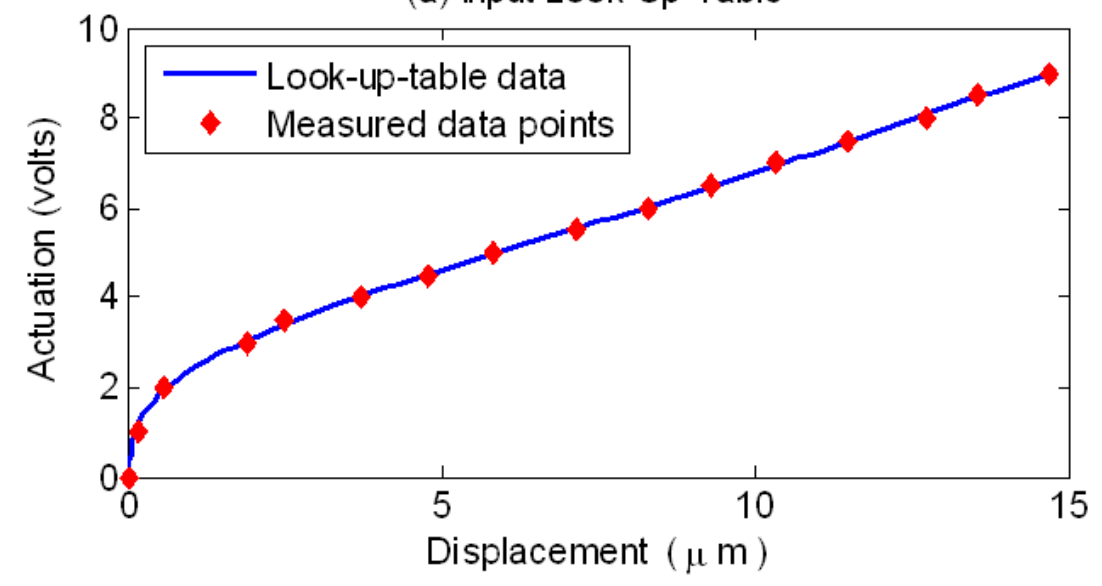

(b) Output Look-Up-Table

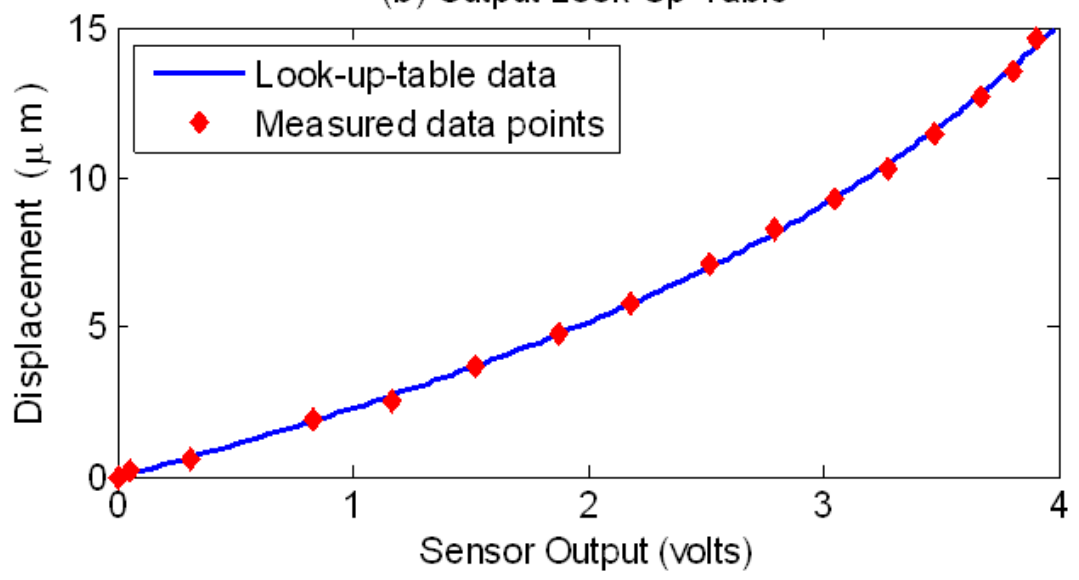

Figure 16 The data used in input and output look-up-tables. [39]

To evaluate the control capability of the open-loop control method, a stair-case reference signal is utilized as shown in Figure 17. The reference voltage increased from 0 to $10 \mathrm{~V}$ with $2.5 \mathrm{~V}$ steps and 50 millisecond time intervals, and then decreased to $0 \mathrm{~V}$ in same manner. Unfortunately, it can been seen in Figure 17 that the open-loop controller cannot provide an acceptable tracking performance for stair-case displacement. A maximum displacement error of $0.96 \mu \mathrm{m}(9.6 \%$ error) was observed at $10 \mu \mathrm{m}$ stroke. This is due to the plant uncertainties and the drift in the sensor and electronic circuits. Plant herein refers to the combination of the electrothermal MEMS device and electronic circuits for actuation and sensing. Therefore, feedback control strategies have to be considered to compensate the uncertainties and errors for accurate positioning. 


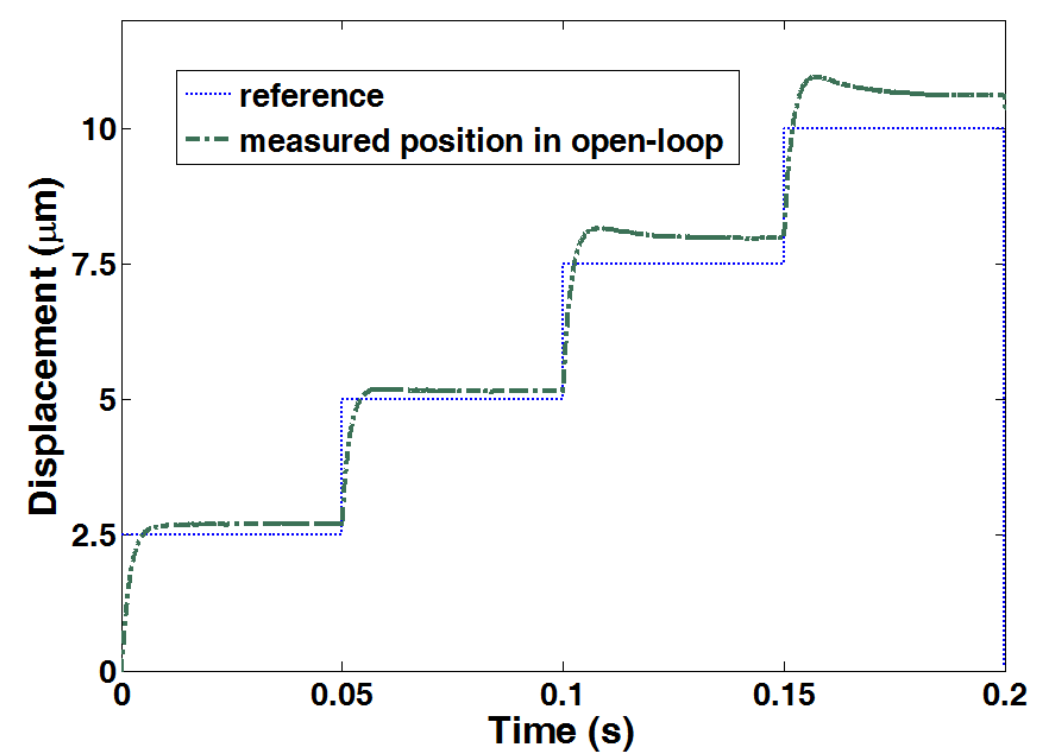

Figure 17 Tracking performance of open-loop control method for a stair case reference. Large over-shoot errors are observed.

\subsection{Closed-loop control}

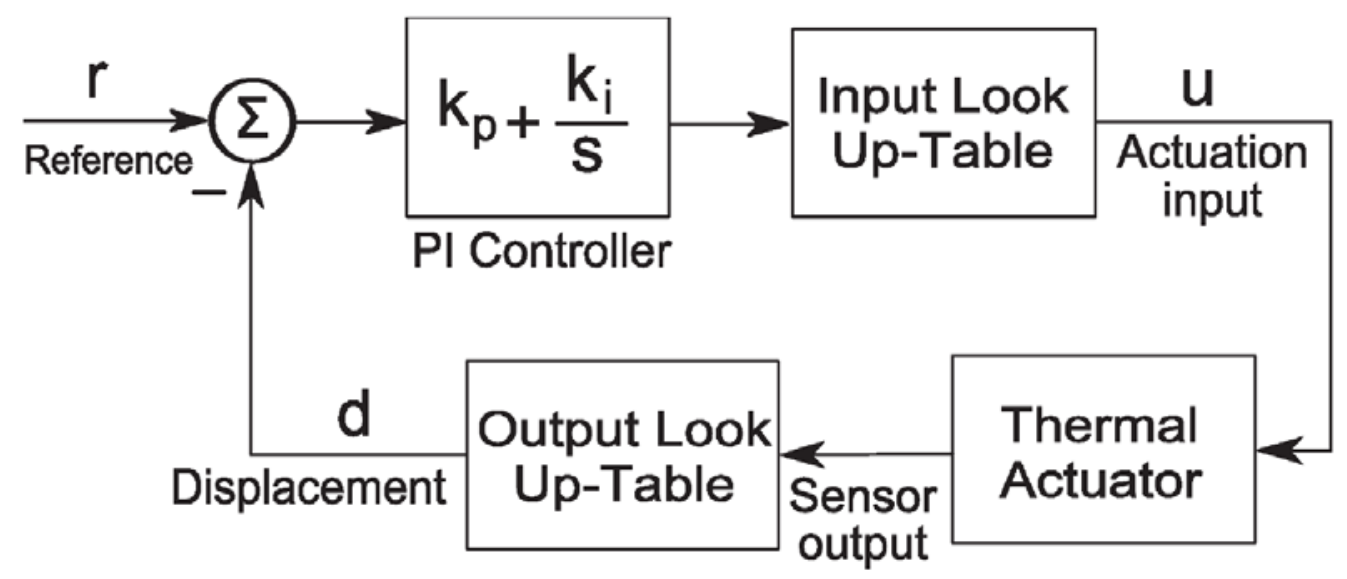

Figure 18 Block diagram of one-degree-of-freedom (1DoF) PI control system including input and output look up tables, reference, electrothermal actuator and sensor, PI controller. [39]

For closed-loop control, we incorporated a proportional-integral controller (PI controller) in addition to the nonlinear mappings, as illustrated in Figure 18. The integration part in the PI controller provides a closed-loop unity low frequency gain form reference to displacement for set point tracking and robustness to uncertainties and disturbances. The proportional part in the PI controller is utilized to reduce the overshoot in step response. The integral gain and proportional gain were determined by experimental measurements. With an 
integral gain of $k_{i}=700$ and a proportional gain of $k_{p}=1.3$, the positioning performance is significantly improved compared to open-loop case, as shown in Figure 19. Based on this control scheme, a controllable desired response of 2.5 $\mu \mathrm{m}$ steps over a $10 \mu \mathrm{m}$ range was obtained with a maximum time constant of 1.4 ms. As illustrated in Figure 19, the overshoot issue in open-loop control was also eliminated.

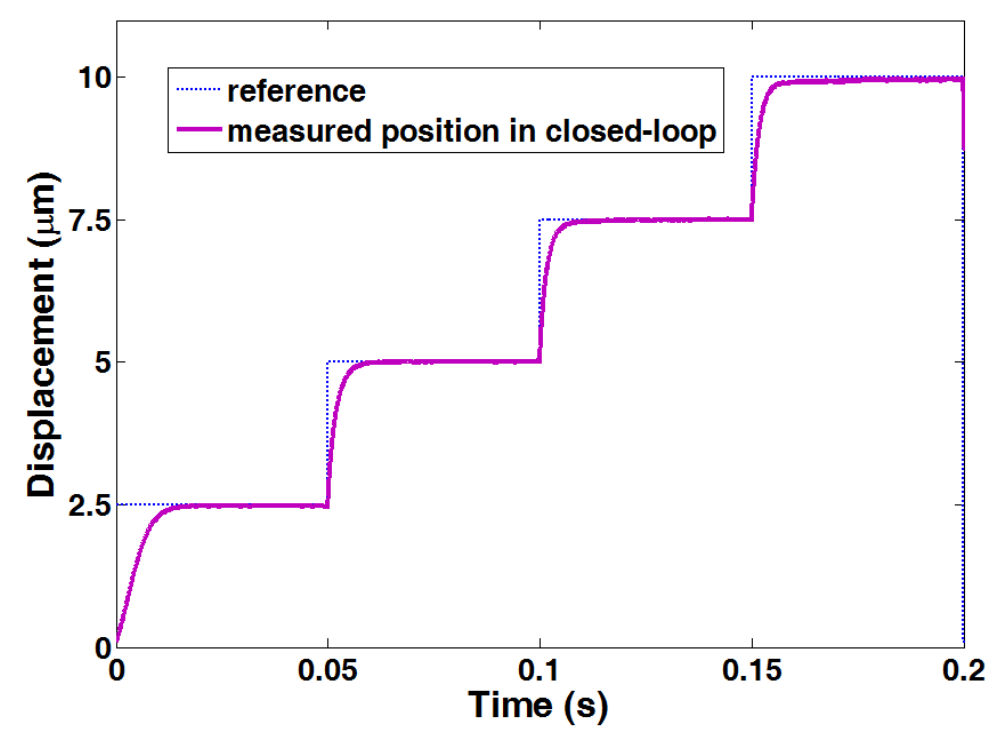

Figure 19 Tracking performance of 1 DoF closed-loop control method for a stair case reference.

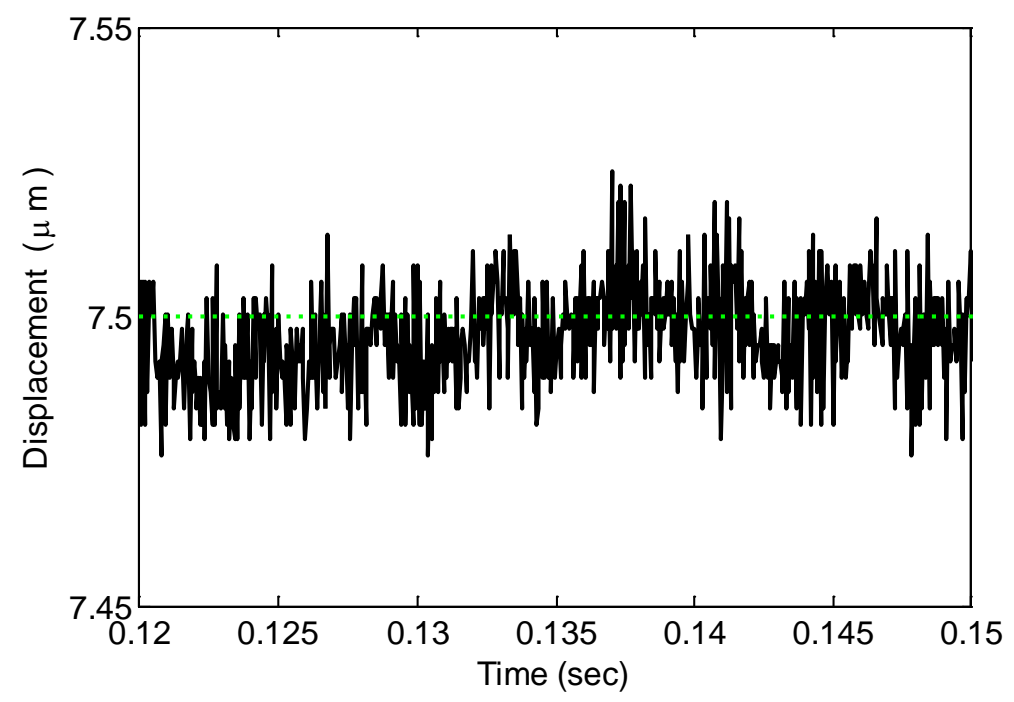

Figure 20 Close-up view of the $7.5 \mu \mathrm{m}$ closed-loop seek operation in Figure 19. RMS positioning error of $8.6 \mathrm{~nm}$ is observed. [39]

Figure 20 is the close-up view of the $7.5 \mu \mathrm{m}$ closed-loop seek operation in Figure 19, which indicates a steady-state RMS positioning error of $8.6 \mathrm{~nm}$. As a 
comparison, the steady-state RMS positioning error of a similar open-loop seek operation can reach to $1180 \mathrm{~nm}$.

To further investigate the tracking capability of the nanopositioner, a $10 \mathrm{~Hz}$ triangular reference covering a $10 \mu \mathrm{m}$ range was applied. The one-degree-offreedom (1DoF) PI control system in Figure 18 yielded an acceptable control performance, as shown in Figure 21 (labeled "No prefilter"). With an integral gain of $k_{i}=2000$ and a proportional gain of $k_{p}=4$, the steady-state tracking error has a standard deviation of $90 \mathrm{~nm}$, which is less than one percent of the $10 \mu \mathrm{m}$ full range.

(a)

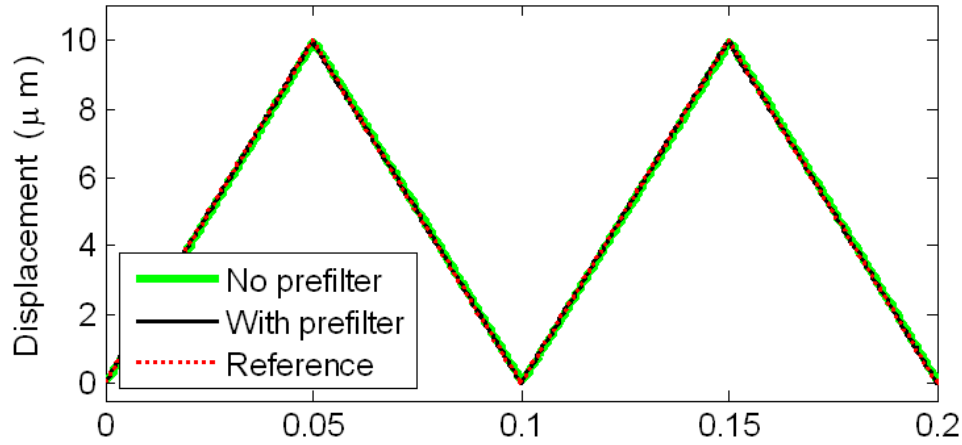

(b)

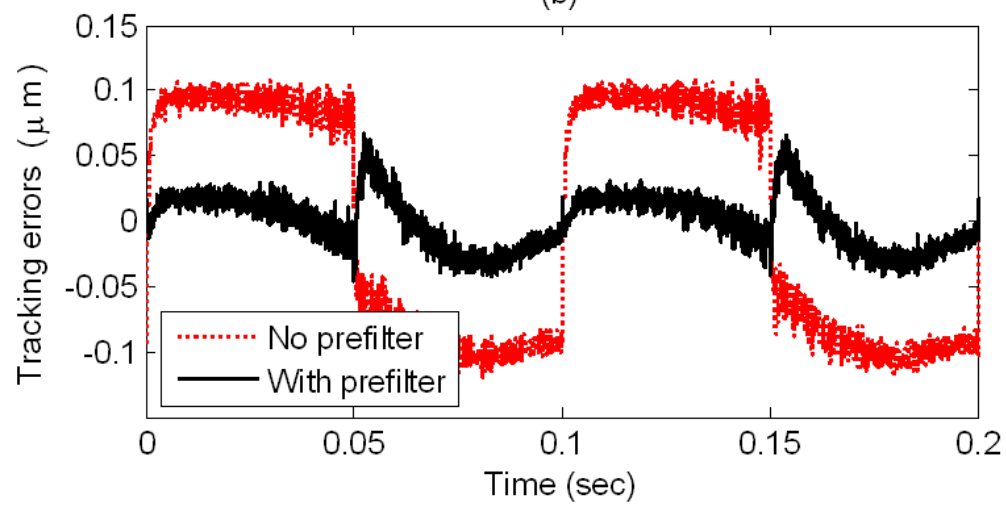

Figure 21 Performance of 1DoF (no prefilter) and 2DoF (with prefilter) control systems in tracking of a triangular reference. RMS positioning error of $90 \mathrm{~nm}$ is observed. [39]

The control performance can be further improved with a 2DoF control system consisting of a PI controller and a prefilter. The look-up-tables provide the nonlinear mappings described earlier. To provide better stability margins we used lower gains of $k_{i}=1100$ and $k_{p}=2$ for the PI controller. This also reduces the effect of measurement noise on the controlled displacement output due to feedback. The tracking performance for a $10 \mathrm{~Hz}$ triangular reference is shown in Figure 21 (labeled "With prefilter"). It can be observed that displacement output closely follows a desired triangular reference within a wide range of $10 \mu \mathrm{m}$ with a standard deviation of $20 \mathrm{~nm}$. 


\section{Conclusion}

The control issues of Microelectromechanical System (MEMS) nanopositioning devices are introduced and discussed. A novel micromachined silicon nanopositioner with on-chip electrothermal actuators and electrothermal sensors has been presented with nanometre resolution and low drift. The MEMS positioner was embedded in a feedback loop to realize a precise position control. Due to the nonlinear nature of the electrothermal actuator, a nonlinear inversion block was added to the feedback loop to linearize the plant. The experimental results showed that the positioner with a PI controller achieved a high degree of positioning accuracy with high robustness.

The electrothermal actuator positions the heat-sink plate, which is a suitable platform for samples located on it as a load. This one-dimensional positioner is a preliminary experimental device for the future two-dimensional positioners [45], which can be used as a nanopositioner stage for scanning probe microscopy. As the forces induced by thermal expansion of the arms are large, it is expected that the performance of the positioner would not significantly change if small probes or samples are attached to the platform. Due to the limitation of the SOIMUMPs fabrication process, it is not possible to design a two-dimensional thermal based positioner in a single device layer. However, the thermal sensors and actuators can be placed in different layers, which can be fabricated in METALMUMPs process through MEMSCAP.

\section{Acknowledgment}

This research is funded by Australian Research Council (ARC) discovery grant- DP0774287 and the Griffith University Research Infrastructure Program fund from Griffith University, Australia. 


\section{References}

[1] G. Binnig and H. Rohrer, "The scanning tunneling microscope,” Scientific American, vol. 253, pp. 50-56, 1986.

[2] G. Binnig, C. Quate and C. Gerber, “Atomic force microscope,” Physical Review Letters, vol. 56, no. 9, pp. 930-933, 1986.

[3] A. Pantazi, A. Sebastian, G. Cherubini, M. Lantz, H. Pozidis, H. Rothuizen and E. Eleftheriou, "Control of MEMS-Based scanning-probe data-storage devices,” IEEE Transactions on Nanotechnology, vol. 15, no. 5, pp. 824841, 2007.

[4] A. Pantazi, M. A. Lantz, G. Cherubini, H. Pozidis and E. Eleftheriou, “A servomechanism for a micro-electro-mechanical-system-based scanningprobe data storage device,” Nanotechonology, vol. 15, no. 10, pp. 612-621, 2004.

[5] G. Binnig, M. Despont, U. Drechsler, W. Häberle, M. Lutwyche, P. Vettiger, H. J. Mamin, B. W. Chui and T. W. Kenny, "Ultrahigh-density atomic force microscopy data storage with erase capability,” Applied Physics Letters, vol. 74, no. 9, p. 1329-1331, 1999.

[6] M. Despont, J. Brugger, U. Drechsler, U. Dürig and W. Häberle, "VLSINEMS chip for parallel AFM data storage," Sensors and Actuators A: Physical, vol. 80, no. 2, p. 100-107, 2000.

[7] P. Vettiger, G. Cross, M. Despont, U. Drechsler, U. Dürig, B. Gotsmann, W. Häberle, M. Lantz, H. Rothuizen, R. Stutz and G. Binnig, “The 'millipede'-Nanotechnology entering data storage," IEEE Transactions on Nanotechnology, vol. 1, no. 1, pp. 39-55, 2002.

[8] Y. K. Yong, S. S. Aphale and S. O. Moheimani, "Design, Identification, and Control of a Flexure-Based XY Stage for Fast Nanoscale Positioning," IEEE Transactions on Nanotechnology, vol. 8, no. 1, pp. 46-54, 2009.

[9] Y. K. Yong, S. S. Aphale and S. O. Moheimani, “Atomic force microscopy with a 12-electrode piezoelectric tube scanner," Review of Scientific Instruments, vol. 81, no. 3, p. 033701, 2010. 
[10] B. J. Kenton and K. K. Leang, "Design and control of a three-axis serialkinematic high-bandwidth nanopositioner," IEEE/ASME Transactions on Mechatronics, vol. 17, no. 2, pp. 356-369, 2012.

[11] S. Schonhardt, J. G. Korvink, J. Mohr, U. Hollenbach and U. Wallrabe, "Optimization of an electromagnetic comb drive actuator," Sensors and Actuators A: Physical, vol. 154, no. 2, pp. 212-217, 2009.

[12] C. H. Kim and Y. K. Kim, "Micro XY-stage using silicon on a glass substrate," Journal of Micromechanics and Microengineering, vol. 12, no. 2, pp. 103-107, 2002.

[13] C. K. Pang, Y. Lu, J. Chen, H. Zhu, J. Yang, J. Mou, J. Guo, B. M. Chen and T. H. Lee, "Design, fabrication, sensor fusion, and control of a micro X-Y stage media platform for probe-based storage systems," Mechatronics, vol. 19, pp. 1158-1168, 2009.

[14] N. Sarkar and R. Mansour, "A CMOS-MEMS Scanning Probe Microscope with Integrated Position Sensors," in 1st Microsystems and Nanoelectronics Research Conference (MNRC 2008), 2008.

[15] N. Sarkar, R. Mansour, O. Patange and K. Trainor, "CMOS-MEMS Atomic Force Microscope,” in 16th International Solid-State Sensors, Actuators, and Microsystems Conference (Transducers'11), 2011.

[16] D. Barrettino, S. Hafizovic, T. Volden, J. Volden, K. Kirstein, A. Hierlemann and H. Baltes, "CMOS Monolithic Atomic Force Microscope," in 2004 Symposium On VLSl Circuits, Digest of Technical Papers, 2004.

[17] S. Devasia, E. Eleftheriou and S. O. Moheimani, “A survey of control issues in nanopositioning," IEEE Transactions on Control Systems Technology, vol. 15, no. 5, pp. 802-823, 2007.

[18] A. Sebastian and D. Wiesmann, "Modeling and experimental identification of silicon microheater dynamics: a systems approach," Journal of Microelectromechanical Systems, vol. 17, no. 4, pp. 911-920, 2008.

[19] Y. C. Chen, R. T. M'Closkey, T. A. Tran and B. Blaes, “A Control and Signal Processing Integrated Circuit for the JPL-Boeing Micromachined Gyroscopes,” IEEE Transactions on Control Systems Technology, vol. 13, no. 2, pp. 286-300, 2005. 
[20] V. Todorov, V. Stavrov and J. Kreuter, "Sub nm-Resolution Static Measurement with MEMS Displacement Sensors,” Procedia Engineering, vol. 25, p. 591-594, 2011.

[21] L. Gu, X. Li, H. Bao, B. Liu, Y. Wang, M. Liu, Z. Yong and B. Cheng, "Single wafer processed nanopositioning XY-stage with trench-sidewall micromaching technology," Journal of Micromechanics and Microengineering, vol. 16, no. 7, pp. 1349-1357, 2006.

[22] C. Lee, S. Han and N. MacDonald, "Single crystal silicon (SCS) XY-stage fabricated by DRIE and IR alignment,” in Proceeding of IEEE MEMS2006, 2006.

[23] A. G. Fowler, A. N. Laskovski, A. C. Hammond and S. O. Moheimani, "A 2-DoF electrostatically actuated MEMS nanopositioner for on-chip AFM," Journal of Microelectromechanical Systems, vol. 21, no. 4, pp. 771-773, 2012.

[24] J. J. Gorman, Y. Kim and N. G. Dagalakis, "Control of MEMS nanopositioners with nano-scale resolution," in Proceeding of IMECE2006, Chicago, 2006.

[25] R. Hickey, D. Sameoto, T. Hubbard and M. Kujath, "Time and frequency response of two-arm micromachined thermal actuators," Journal of Micromechanics and.Microengineering, vol. 13, no. 1, pp. 40-46, 2003.

[26] Y. Sun, M. A. Greminger, D. P. Potasek and B. J. Nelson, “A Visually Servoed MEMS Manipulator," in Experimental Robotics VIII, Berlin/Heidelberg, Springer, 2003, pp. 255-264.

[27] L. L. Chu and Y. B. Gianchandani, "A micromachined 2D positioner with electrothermal actuation and sub-nanometer capacitive sensing," Journal of Micromechanics and Microengineering, vol. 13, no. 2, pp. 279-285, 2003.

[28] Y. S. Choi, Y. Zhang and D. W. Lee, "A thermal-driven silicon micro xystage integrated with piezoresistive sensors for nanopositioning," Journal of Micromechanics and Microengineering, vol. 22, no. 5, p. 055002, 2012.

[29] L. Sun, J. Wang, W. Rong, X. Li and H. Bao, "A silicon integrated micro nano-positioning XY-stage for nano-manipulation," Journal of Micromechanics and Microengineering, vol. 18, no. 12, p. 125004, 2008. 
[30] L. Ji, Y. Zhu, S. O. Moheimani and M. R. Yuce, “A micromachined 2DOF nanopositioner with integrated capacitive displacement sensor," in Sensors, 2010 IEEE , Hawai, 2010.

[31] M. A. Lantz, G. K. Binnig, M. Despont and U. Drechsler, "A micromechanical thermal displacement sensor with nanometre resolution," Nanotechnology, vol. 16, no. 8, pp. 1089-1094, 2005.

[32] G. K. Binnig, M. Despont, M. A. Lantz and P. Vettiger, "Thermal movement sensor”. International Patent WO 2004/020328 A1.

[33] N. B. Hubbard, M. L. Culpeper and L. L. Howell, "Actuators for micropositioners and nanopositioners," Applied Mechanics Reviews, Transactions of the ASME, vol. 59, no. 6, pp. 324-334, 2006.

[34] Y. Zhu, S. O. Moheimani and M. R. Yuce, "Simultaneous capacitive and electrothermal position sensing in a micromachined nanopositioner," IEEE Electron Device Letters, vol. 32, no. 8, pp. 1146-1148, 2011.

[35] Y. Zhu, A. Bazaei, S. O. Moheimani and M. R. Yuce, "A micromachined nanopositioner with on-chip electrothermal actuation and sensing," IEEE Electron Device Letters, vol. 31, no. 10, pp. 1161-1163, 2010.

[36] A. Sebastian, A. Pantazi, S. R. Moheimani, H. Pozidis and E. Eleftheriou, "Achieving subnanometer precision in a MEMS-based storage device during self-servo write process," IEEE Tran. on nanotechnology, vol. 7, no. 5, pp. 586-595, 2008.

[37] M. A. Lantz, H. E. Rothuizen, U. Drechsler, W. Haberle and M. Despont, "A vibration resistant nanopostioner for mobile parallel-probe stroage applications,” Journal of Microelectromechanical Systems, vol. 16, no. 1, pp. 130-139, 2007.

[38] V. Kaajakari, Practical MEMS, Las Vegas: NV: Small Gear Publishing, 2009.

[39] Y. Zhu, A. Bazaei, S. R. Moheimani and M. R. Yuce, “Design, Modeling, and Control of a Micromachined Nanopositioner With Integrated Electrothermal Actuation and Sensing," Journal of Microelectromechanical Systems, vol. 20, no. 3, pp. 711-719, 2011. 
[40] A. Bazaei, Y. Zhu, R. Moheimani and M. R. Yuce, “Analysis of Nonlinear Phenomena in a Thermal Micro-Actuator with a Built-In Thermal Position Sensor,” IEEE Sensors Journal, vol. 12, no. 6, pp. 1772-1784, 2012.

[41] [Online]. Available: http://www.memscap.com/en_mumps.html .

[42] Software Manual - Planar Motion Analyzer Software 2.3, Polytec.

[43] U. Durig, "Fundametals of micromechanical thermoelectric sensors," Journal of Applied Physics, vol. 98, p. 044906, 2005.

[44] C. D. Lott, T. W. McLain, J. N. Harb and L. L. Howell, "Modeling the thermal behavior of a surface-micromachined linear-displacement thermomechanical microactuator," Sensors and Actuators A, vol. 101, no. 2002, pp. 239-250.

[45] M. Rakotondrabe, A. G. Fowler and S. R. Moheimani, "Control of a Novel 2-DoF MEMS Nanopositioner With Electrothermal Actuation and Sensing," IEEE Transactions on Control Systems Technology, vol. 22, no. 4, pp. 1486-1497, 2014. 